\title{
A Pilot Study for Utilizing Additive Manufacturing and Responsive Rewards in Physical Activity Gamification
}

\author{
James I. Novak and Jennifer Loy
}

Product Design and Design for Additive Manufacturing, University of Technology Sydney, Australia

James.Novak@uts.edu.au

\begin{abstract}
Health related issues from being overweight or obese are significant global challenges, and whilst increased activity is known to reduce the health risks associated with these conditions, current wearable and activity tracking devices alone are insufficient to motivate everyone over the long-term necessary to make significant change. This paper explores novel gamified systems as part of a pilot study to leverage additive manufacturing and Internet of Things technologies to increase motivation for physical activity, creating new ways for people to be rewarded in the physical world, and for activity data to be communicated in more abstract and customisable ways. These systems were exhibited and discussed at the 2017 Design 4 Health conference in Melbourne, Australia, and are intended to contribute to research by designers and fitness companies in thinking beyond the digital interface, and in particular to engage young people in the physical world.
\end{abstract}

Keywords: health; human-centred design; internet of things; product design; obesity 


\section{Introduction}

Overweight and obesity are defined as abnormal or excessive fat accumulation that presents a risk to health... Overweight and obesity are major risk factors for a number of chronic diseases, including diabetes, cardiovascular diseases and cancer. Once considered a problem only in high income countries, overweight and obesity are now dramatically on the rise in low- and middle-income countries, particularly in urban settings. (World Health Organisation 2018)

According to the most recent data provided by the World Health Organisation (2018), more than 1.9 billion adults aged over eighteen years old or over are overweight globally, with $13 \%$ falling within the obese category. In addition, it is estimated that over 380 million children would have been identified as overweight or obese in 2016. As obesity is considered preventable, a cultural shift in thinking is required to change behaviours from a young age and though into adulthood.

As part of a response to this issue, Human-Computer Interaction (HCI) designers have developed personal activity trackers which may be worn on the wrist (for example Fitbit), waist (more traditional pedometers or mobile phones with fitness applications) or inside a piece of clothing like shoes (such as the Nike+ or Adidas miCoach). A personal activity tracker can be defined as a device that is worn on the body, uses sensors such as accelerometers to track the user, and connects this data to an online database to show trends over time (Hoy 2016). Such activity trackers have experienced significant market growth over recent years (Gouveia, Barros, and Karapanos 2014), allowing people to automate the tracking of physical activities, for example number of steps, energy expended, distance traversed, heart rate, and quality of sleep. These mobile and wearable systems may be datacentric in their accurate numerical display of data to the user, or employ game-like qualities with the assumption that by applying game design principles (gamification) to physical 
activity, the activities will become more enjoyable and therefore more likely to be repeated and improved upon (Zuckerman and Gal-Oz 2014). Nicholson (2012) explains:

A common implementation of gamification is to take the scoring elements of video games, such as points, levels, and achievements, and apply them to a work or educational context. While the term is relatively new, the concept has been around for some time through loyalty systems like frequent flyer miles, green stamps, and library summer reading programs. (Nicholson 2012)

In order for gamified methods of activity tracking to succeed in their intent to increase physical activity in users, they need to be based on human-centred design principles. However, recent evidence suggests that activity tracking devices often only improve physical activity during the short-term and there is a lack of supporting data on their ability to improve the health and fitness of individuals over the long-term (Ledger and McCaffrey 2014). Furthermore, peer reviewed studies into gamification and exercise have only considered an adult market, and this study presents new concepts specifically addressing the needs of a younger market aged 12-18 years of age. Long-term engagement will be necessary to support a culture shift sufficient to impact the current obesity epidemic, and ensure the next generation of adults have suitable tools to ensure a healthy lifestyle.

The aim of this research was to explore the technical possibilities of new responsive systems that leverage developments in digital technology, in particular additive manufacturing and the Internet of Things. Through experimental design and prototyping, this article argues it is possible to develop new tangible reward systems that augment the reliance of current devices upon interfaces as the principle means of connection to the gamified elements of activity tracking. One of the examples of this pilot study is a functional prototype featured in an exhibition at the 2017 Design4Health conference in Melbourne, Australia, as pictured in Figure 1 (Novak and Loy 2017a). The examples show how product designers are 
beginning to embrace new opportunities for products to co-exist in the physical and digital worlds, capable of responding to changing human needs over time. This is known as 4D (four dimensional) product design (Novak and Loy 2017b). The research supporting this article considers tracking technology beyond the digital interface, exploring emerging opportunities to engage particularly with young people in the physical world.

Figure 1. 'Metamorphosis' interactive prototype exhibited at Design4Health2017, Melbourne, Australia

\section{Background}

Despite good intentions, numerous studies have shown that the use of wearable activity trackers is not sufficient to engage with all users over the long-term; a recent study found that a third of consumers in the United States stopped using their device within six months of receiving it (Ledger and McCaffrey 2014), whilst from a medical perspective, there is a lack of empirical evidence supporting the use of activity trackers to improve patient health (Gouveia, Barros, and Karapanos 2014; Piwek et al. 2016).

The bad news is that this industry has reached a subtle but important plateau. Despite our ability to gather more physiological data than we have been able to in the past with the wide range of new sensors we're seeing on devices (heart rate, galvanic skin response, temperature, etc.), we haven't yet figured out how to robustly translate this data into meaningful insights for users, outside of very narrow use cases. (Ledger 2016,9)

Don Norman, whose research focuses on human-centred design, argues that this is because 'much of what is being done is happening simply because it can be done' (2013), with new technologies often embedded within wearable and mobile devices without being driven by genuine need or consumer pull. Increased capacity for devices to sense the real-world has led 
to a 'gizmo' culture (Sterling 2005), where increasing device features has become more important than simple, well-considered design solutions to genuine human problems. While activity tracking lacks universal engagement with users, it is important to highlight that many people are motivated by the social, competitive, and virtual aspects of activity tracking, with popular platform Strava having its one billionth activity uploaded in 2017 (Strava 2017). However, as new technologies emerge, and digitally immersed generations mature, new ways for younger users transitioning to adulthood to be encouraged to maintain a physically active lifestyle will need to be developed (Baranowski and Frankel 2012).

Much like the mixed research around the effects of activity trackers on improving health across all user groups, similar uncertainty exists around the use of gamification to improve engagement with physical activity. Zuckerman and Gal-Oz $(2014,1717)$ found through extensive analysis of literature that 'due to contradicting findings from prior studies, and lack of systematic research in the field, [the assumption that gamification increases the motivation to perform physical exercise] cannot be supported by the existing literature.' Hamari, Koivisto and Sarsa (2014) conducted a similarly broad literature review with more favourable results for the use of gamification, however, 'the effects are greatly dependent on the context in which the gamification is being implemented, as well as on the users using it' (Hamari, Koivisto, and Sarsa 2014, 3025). Overall, based on these broad literature assessments, the effects of gamification appear to be mixed and highly dependent upon the individual user and context. Research suggests that extrinsic motivators like games and rewards 'should be viewed primarily as a temporary motivating tool and should be used with caution by practitioners attempting to promote activity' (Kilpatrick, Herbert and Jacobsen 2002 , 41). For many, the initial novelty of gamified systems wears off, and a lack of intrinsic motivation results in a return to insufficient physical activity. Therefore, new extrinsic 
motivators are needed to better fulfil the temporary need to provide motivation for some people, and novel research studies have been experimenting with these for many years.

Fish'n'Steps (Lin et al. 2006) is an example of a novel gamified system for encouraging increased step count, with users being assigned a virtual pet fish within a virtual tank, along with a pedometer. As users meet and exceed their daily goals, their pet fish grows, even spawning baby fish as goals are consistently exceeded. However, when goals are not met, the fish becomes physically smaller, and will change facial expressions from a happy to a sad face. Only $53 \%$ of the nineteen participants in the study sustained daily interactions with their pet fish for the duration of the fourteen-week study and, while the authors note that some participants reported emotionally bonding with their fish, other participants were more motivated by the competition amongst participants than the fish itself. It was also found that 74\% of participants increased their steps from their baseline using the Fish'n'Steps system, however, inversely this also means $26 \%$ of people were not motivated to perform extra activity at all. As a relatively small study, the results are not statistically significant; however, they do provide insight into the use of unrelated novelty as extrinsic motivation, which is not directly linked to the underlying intent itself, with some positive results.

Similar research has been performed in a project called Ubifit (Consolvo et al. 2008), featuring a virtual garden displayed as the wallpaper on a mobile phone, with the garden being a metaphor for the user's physical activity. This can be seen as having a more direct psychological relationship to the use in terms of perceived health benefits. Flowers bloom and butterflies emerge as the user achieves certain goals, or the garden become sparse and covered mostly with grass if goals are not achieved, focusing on positive reinforcement rather than punishing the user with wilting flowers and exposed soil. Overall the users found the metaphor to be enjoyable, with the real concerns being about the accuracy of the system and need to frequently manually modify or enter information that the software did not 
automatically recognise as a fitness activity, or that it inferred as an incorrect activity. This was a very short pilot study of three weeks duration, with only twelve participants, so there is limited understanding of the long-term engagement of such a gamified system, or how a broad cross-section of the population responds.

One of the challenges for commercial and research systems is that the rewards for adults and older children are intangible, usually taking the form of digital badges, trophies or other virtual achievements, which only exist within the application or software being used. Studies have shown mixed results from these virtual forms of reward (Zuckerman and GalOz 2014), with customization of rewards by users suggested by Nicholson (2012) as one way to enhance meaning and motivation within gamified systems. While many activity trackers permit some level of customization, for example a choice of daily step goal or visual screen display, these are limited and not likely to engage all users over the long-term. Primary research provided further insight into some of these factors, with one of the authors using a Garmin Vivofit wrist-worn activity tracker as part of a review of the technology for designled research. Although of no statistical research value, this experience provided the authors with personal insight into the psychological impact of the trackers that informed the direction of the human-centred design approach discussed later in the article.

The data collected by the author is shown in Figure 2, with a steady decline in monthly achievements until the device stopped being used after six months, aligning with the reported statistic of one third of users who stop using an activity tracker within the first six months (Ledger and McCaffrey 2014).

Figure 2. Graph showing percentage of monthly goals with Garmin Vivofit 2014-2015 
This experience provided the basis for problem-framing for the following human-centred design issues:

(1) The activity tracker was itself an interface and required the use of additional interfaces (computer or mobile phone) to upload data, analyse trends over time, and achieve virtual rewards. The human in this relationship began serving the computer, rather than having it be of service, which contradicts the second principle of Krishna's (2015) call to move beyond interfaces, and leverage computers instead of serving them. The simple act of walking or sleeping became a chore.

(2) The device required constant attention: regular syncing to another interface, performing software updates, holding a button to record the time of going to sleep, and observation of the visual alerts which increased every fifteen minutes to remind the wearer to take a break and do some physical activity. It was a true 'gizmo' (Sterling 2005) and did not blend into the background of daily life.

(3) The activity tracker lacked any understanding about the user; for example, after spending an afternoon kitesurfing (which the device could not track) it would still continue displaying reminders to be more active and go for a walk. With steps being its only metric, it was extremely limited in its definition of 'activity.'

(4) The challenges presented to adults would be amplified for a younger demographic.

Furthermore, the Garmin Vivofit only displays basic numerical data on its interface; the process to view more graphic displays and analyse long-term patterns is convoluted requiring the following process:

(1) Turn on the computer.

(2) Wait for it to load.

(3) Log in to the computer account. 
(4) Wait for it to load.

(5) Find and open the Garmin Express software (assuming it is already installed). If using a different computer, the user will need to download and install the software, and connect it to their account.

(6) Wait for the software to load.

(7) Connect the ANT+ USB to the computer (the USB is very small, which in itself can create a problem for users when it is misplaced).

(8) Hold the button on the Garmin Vivofit for 2 seconds until 'sync' appears on its screen. Do not hold it for longer than 2 seconds or the Vivofit will go to sleep. Do not hold it for less than 2 seconds or the screen will cycle to the next data display.

(9) Wait for the data to sync to the Garmin Express software.

(10) Click on the Garmin Connect icon to open the Garmin Connect web portal.

(11) Wait for the web page to load and refresh with the newly added data.

(12) View the data.

(13) Manually make changes to any data that is incorrect e.g. what time did the user really go to bed three days ago? What sort of activity was happening between 2:15-2:45pm the day before: running, cycling, cleaning? What shoes were worn during this activity? Does the user want to share this data on social media?

This thirteen step process and the significant time required to view basic data about relatively mundane activity is similar to an example given by Krishna (2015) about the complex process for unlocking a BMW car with a mobile app, which also took 13 steps and was far more complex than using a physical key. These are both examples of 'complexification' (Greenfield 2006), needlessly overcomplicating simple daily tasks with the addition of an interface and requirement to mediate between the computer and reality. The user experience is almost entirely spent in service of the computer system, rather than leveraging the power of 
computers and sensors to simply perform these tedious processes in the background of daily life, for example whilst working at the computer on other activities. The system provided virtual reward badges that were quickly earned during the first months but then not customisable to advance individual goals. This included a virtual badge for achieving two million steps, which is a goal without any real context or meaning for the user, and rarely the sort of goal a person would hope to achieve, as compared to something more tangible like running a marathon. Newer incarnations of activity trackers make syncing and analysing data more accessible through a Bluetooth connection to a mobile phone and smart watches are capable of collecting and displaying data natively. However, these systems remain reliant upon low-level data visualizations and virtual rewards to motivate activity. This is a plateauing (Ledger 2016) in their development that is simultaneously plateauing their ability to influence behaviour and attitudes, and therefore constraining their ability to impact the growth in obesity and health-related problems in society. As a result of this primary and secondary research, a series of prototypes were developed to attempt to break the reliance of activity tracking systems on interfaces, and propose new relationships between people, their activity, and the ways they receive information about their achievements.

\section{Responsive Systems}

While there is evidence both supporting and refuting claims about the effectiveness of rewards for motivation (Zuckerman and Gal-Oz 2014), Nicholson (2012) suggests the customization of goals and rewards as critical to the success of gamified systems. The same link between customization and enhanced customer engagement can be seen in product design with the rise in additive manufacturing (3D printing). This is a significant fabrication technology that has recently evolved from a prototyping technology, to one where end-use materials and processes can be used to create parts without the traditional investment in 
tooling required for manufacturing. This allows for the cost-effective production of individual objects which can be personalized to 'provide more comfort, unique aesthetic appeal, or better performance' (Shugrina, Shamir, and Matusik 2015) than generic mass-manufactured products can currently achieve. As 3D printers become increasingly accessible, researchers suggest consumers will shift to prosumers (Ahluwalia and Miller 2014; Fleischmann 2015) who both produce and consume products enabled by digital manufacturing technologies. This opens new opportunities to imagine the relationship between gamification, data collection and physical activity, and led to the development of prototypes to explore new ways to provide extrinsic motivation to younger users who are growing up in an increasingly connected digital world, and will require new solutions to engage them in physical activity beyond the ubiquitous interface they have grown up with. With 3D printers proliferating schools (Horejsi 2014; Wilson 2013), Makerspaces on the rise in the community (Lou 2016), and online service bureaus like Shapeways (www.shapeways.com) and i.Materialise (www.i.materialise.com) providing worldwide access to high-end materials, design and 3D printing skills will become core to the next generation, and digital platforms like activity tracking will need to respond.

\section{Example 1: Parametric Change}

By linking 3D Computer-Aided Design (CAD) files to the data generated by an activity tracker, a responsive parametric system can convert raw activity data into custom 3D CAD models suitable for 3D printing. Parametric design systems use relationships to drive their geometry, with 'each active variable caus[ing] the overall system to change its behaviour and thereby generate variations without losing the overall coherence and integrity of the system' (Qian 2009, 22). In an appropriately designed parametric system, changes can occur ad nauseam without detrimental impact on the three dimensional model or the ability to 3D print the model. In the research informing this article, the proposition was that tangible rewards 
produced through 3D printing could symbolise levels of achievement in more novel and bespoke ways than the raw Garmin data and graphs, allowing the user to project their own meaning onto the outcomes and connect with the results in a more physical way. This geometry could be personalized to suit the profile of the user. For example, in a design aimed at engaging the youngest appropriate uses in the materialization of their achievements using 3D printing, initial experiments featuring a cartoon bear character were developed, as shown in Figure 3, with the bear in this example changing size and colour based on the data from Figure 2. This algorithm is generated using Rhinoceros CAD software with the Grasshopper plugin, and allows any 3D model, such as those downloaded freely from online platforms like Thingiverse (www.thingiverse.com), to be plugged in to the system. This initial proof-ofconcept led to a more sophisticated example involving greater flexibility and communication of user results.

Figure 3. Monthly Garmin percentage of goal data turned into 3D models (December on the left to May on the right)

\section{Metaphor Development}

Metamorphosis, being the transition of something from one form to another, was selected as a metaphor to explore in the further development of this project for children to symbolise achievement, with research suggesting users respond well to metaphorical relationships to data and ideas of growth and change over time (Consolvo et al. 2008). The metamorphosis of a caterpillar into a butterfly was chosen to address issues relating to the negative connotations of raw data and graphs, which are more explicit in their display of failed goals. In this project (called Metamorphosis) a caterpillar, consisting of a head and body modules, grows in relation to the automated daily/weekly/monthly goals, with each body module representing a 
$10 \%$ increment towards the goal. Once a user achieves $100 \%$ of their goal (10 body modules), a butterfly replaces the caterpillar and increases in size based on the percentage of goal over $100 \%$. By using percentages rather than the actual steps, this system allows for the goals to fluctuate over time without affecting the system. Using the data captured with the Garmin Vivofit during 2014-2015, the 3D models were automatically generated using Rhinoceros and Grasshopper. These examples were 3D printed on a desktop FusedDeposition Modelling (FDM) machine as shown in Figure 4 for the 2017 Design4Health conference exhibition held in Melbourne, Australia.

Figure 4. 3D printed models of December-May

Unlike the negative connotations experienced by participants in the Fish'n'Steps study where the negative results were depicted with a small, sad fish, when these 3D printed models are viewed without reference to the data their meaning is less disheartening for months where goals are not achieved. The caterpillar is a colourful, playful form that, whilst grown out of data, does not directly confront the viewer as being related to activity or intrinsic perception of success and failure. Through such abstraction, it is possible for the viewer to create their own meaning related to the object, allowing engagement on a deeper level related to storytelling rather than simple pass/fail metrics. While the metamorphosis of a caterpillar to butterfly was used for this exhibition, co-design could be used in future studies to create any variety of objects suitable for motivating an individual. The approach for the future is not that the designer would create outputs for the user, but rather the designer would create the interface that allows the younger generation to design their own personalized outputs. This approach builds on the digital immersion of younger generations and their growing 
experience of working with 3D printers in the classroom. The millennial generation and those following are better equipped to engage with making and adapting as part of this approach than the current adult population generally are. Storytelling is recognised as being influential upon changing health-related behaviour (Hinyard and Kreuter 2007), particularly for children (Baranowski and Frankel 2012), and is a common element of games and education. However, it is often overlooked in gamified health tracking and motivational systems which often attempt to overlay low-level game-like elements, such as trophies and scoring, onto systems that are designed separately to collect and display data. This results in a disconnect between both elements with an emphasis on static outcomes rather than evolutionary ones. The value of a responsive system, such as prototyped for the Design 4 Health exhibition, is in its potential to better engage young people with their health and relationship with technology.

\section{Materializing the Digital}

The value of this pilot study is in the system that materializes data about physical activity through the use of algorithms and parametric CAD. This is represented in Figure 5 with people engaged in physical activity, which is digitally sensed and communicated wirelessly via the Internet, which manipulates 3D CAD data, then returns to the physical world via 3D printing or other digital manufacturing technology. It is a cyclical process that requires new tools and ways of thinking for designers, with one of the current limitations experienced during this project being the access to secured Garmin data, which could only be manually downloaded in CSV format rather than accessed in real-time by non-Garmin devices or software like Grasshopper. As a result, this process is currently disjointed and not completely automated, and for the Design 4 Health exhibition required a variation to the system whereby a keypad (shown between the 3D prints in Figure 1) allowed attendees to manually enter their own data. However, it is theoretically possible if using a more open activity tracker or 
custom-built mobile application, with Grasshopper and other CAD software capable of accessing online databases or communicating directly with devices.

Figure 5. Cyclical process of sensing the physical world, modifying digital CAD data, and returning the design to the physical world through digital manufacturing

It is important to clarify that while it would be possible to 3D print the results of activity after each day, this is impractical and an irresponsible use of plastic material, even as new biodegradable materials, or recycling systems such as Filabot (www.filabot.com), emerge. As shown at the Design4Health exhibition, it is possible to watch the caterpillar grow in a virtual environment as data changes, and a system may only $3 \mathrm{D}$ print results at the end of a month. Both the virtual and physical representations can provide an insight into how a user is progressing in their goals, without the need to rely on quantified data. Further studies will better clarify suitable time scales for 3D printing results, and the effect on younger users' long-term engagement with physical activity.

\section{Example 2: PEZometer}

Although 3D printing is a significant enabler for the development of personalized systems, responsive rewards are not dependant on this technology. Rather, the argument of this paper is that novel human-computer interactions are needed to engage young people in particular in activity and interest them over the long-term, and through increasingly interconnected cyberphysical systems, new research opportunities are available. The Internet of Things makes possible new product approaches that can replace the digital interface as the default method for communicating the status of physical activity. The design-led research informing this article has been driven by two goals in relation to this proposition: 
(1) To explore a more direct interaction between human and object, rather than visualizing a 3D design on-screen and waiting for it to 3D print (which is currently a very manual and time-intensive process).

(2) Explore the potential of an edible reward system as a challenge to the plastic-based Metamorphosis while responding to the same fitness goals.

3D printed plastic rewards contradict sustainability imperatives, however chocolate and other forms of food printing present the possibility to consider rewards that can safely be disposed of or consumed. This opens up new opportunities that are beginning to emerge with increasingly affordable and accessible 3D printers, ubiquitous mobile and wearable devices, and wireless communication between devices that allows for constant data transmission and sharing. This second pilot project seeks to develop a custom reward system that shifts away from 3D printing to further demonstrate how new physical systems may be used to communicate physical activity achievements beyond the interface.

In order to collect and control the communication of activity data, a custom mobile phone application for Android was developed using MIT App Inventor, allowing collection of pedometer data and manual setting of goals as shown in Figure 6. While the mobile phone is an interface, it is an accessible tool which allows communication over Bluetooth or wireless internet and was not intended to perform any function other than simply setting a goal and performing the computation necessary for the example prototype shown here.

Figure 6. Custom built pedometer mobile phone application Accompanying this mobile phone application was an Arduino Uno microcontroller with Bluetooth receiver. When the user achieved their activity goal, a signal was automatically 
sent via Bluetooth from mobile phone to Arduino, actuating a response automatically without the user needing to access the app or even take their phone from their pocket. This aligns with Krishna's (2015) call for human-computer interactions to shift away from their reliance on interfaces as a move towards good practice in design. Krishna suggests that interfaces will still be useful, but only in limited circumstances, for example, initial setup of a system or other functions that are not critical to the regular operation of the system. In the case described here, the mobile interface is leveraged because of its ubiquity and needs only to be accessed for the setting of a step goal or to view progress (although setting goals could be automated if developed for commercial applications, similar to Garmin). Such a system has been described as 'calm technology' (Weiser and Seely Brown 1996), remaining in the periphery unless action is required.

In order to dispense an edible reward, this project built upon the PEZ candy dispenser due to its simple mechanical operation and the opportunity for users to potentially choose from a multitude of characters, customizing their experience and allowing them to develop stories related to their favourite characters. A small modification was made to the back of the head to attach a wire and the PEZ dispenser sits within a 3D printed sheath, allowing it to be mounted onto a custom plywood enclosure without further modification. It therefore becomes straight forward to swap characters as desired by the user, even in this prototype system. The enclosure houses the Arduino, Bluetooth sensor, batteries, and micro servo which is attached to the other end of the wire and controls the opening movement of the PEZ dispenser. Much like an electronic lock, the PEZ dispenser is only operable by the app, and upon receiving a Bluetooth signal that the step goal has been achieved, will open the PEZ dispenser and expose a single piece of PEZ candy; a small treat that will not upset a health regime. The open and closed states of the prototype are shown in Figure 7, and the prototype has been called a PEZometer (PEZ-pedometer). In this way the candy is not only a reward, but a 
physical signal that goals have been achieved without needing to look at the step data on the phone.

Figure 7. PEZometer in its closed and open states

Like Metamorphosis, the PEZometer is enabled by the increasingly blurred boundaries between the physical and digital worlds and re-imagines the way people can be rewarded for achieving physical activity goals. These prototypes illustrate that designers are no longer limited to developing static products and systems that are identical for all users, which was a consequence of the industrial revolution and shift to mass production. Rather, this research signals a shift towards methods of engaging individual people in physical activity beyond the interface. This is particularly relevant for the younger generation as it responds not only to the megatrend of digital immersion identified by Hajkowicz (2015), but also of their increased expectations in terms of personalized experiences. Further research and user testing is required to quantify the effects physical reward systems may have on the long-term physical activity of people; however, the suggestion in this article is that data can be used to drive more responsive, real-world interactions with young people that allow for greater customization and the opportunity for people to attach their own meaning and stories to their activity to improve the long-term impact of these systems on changing behaviour.

While 3D printing is still a relatively slow process, and the production of rewards would require automation to occur while the user is at work or sleeping, the PEZometer is capable of dispensing rewards immediately as goals are achieved in real-time, linked to smart phones which people already carry with them without requiring a separate activity tracking wearable device. In Greenfield's $(2006,92)$ discussion of ubiquitous computing and the IoT, 
he describes that 'all the necessary pieces of the puzzle are sitting there on the tabletop, waiting for us to pick them up and put them together;' designers must now play a pivotal role in developing new human-centred products and systems to encourage physical activity, placing people ahead of technology. It is only by fitting around a person's daily life, rather than requiring them to fit around a novel technology (as in the case of the Garmin Vivofit), that significant changes will occur to halt, or even reverse, the obesity epidemic.

\section{Future Research}

Based on successful development of several prototypes, the next stages of this research will partner with healthcare professionals to develop trials with 12-18 year olds to quantify the long-term engagement in physical activity provided by responsive systems. Through collaboration between designers, healthcare professionals and software developers, more robust prototypes and systems will allow products to be deployed in the field for several months, with the most likely method of providing tangible rewards being the use of local 3D printing hubs, such as Makerspaces located in libraries or schools, or a central university hub which can coordinate and quality control the production of rewards and post them to participants. Products connected to each other and the Internet need to be developed to rapidly respond to tracking data, with their technology blended into the background of daily life more effectively, rather than requiring constant attention as is the case at this time. Such responsive systems could help shift activity tracking beyond the current plateau and, considering the cultural shifts in thinking required for the success of the broader imperatives, product designers need to work more effectively in this connected space with healthcare practitioners and researchers to guide this shift based on human-centred design principles.

\section{Conclusion}

The aims of the research informing this article have been to identify the current state of 
activity tracking and gamification and develop examples of functional prototypes that reimagine the relationship people have to activity data. Through this process, human-centred design has been explored through both theoretical and practical research, and the digital interface questioned as the sole means of interaction between people and their activity data. This research responds to the limited success current gamification principles have in engaging users in activities over the long-term. In particular, it highlights the lack of customization for the individual involved in current systems with detachment to generic, virtual rewards as a significant factor that contributes to declining user engagement. Given the significance of obesity issues and the multitude of health risks associated with a lack of physical activity, developing new ways to engage young people in activity is an important challenge that requires new creative thinking, and a focus on human-centred design in collaborations between product designers and healthcare practitioners.

The opportunities provided by recent advances in integrated digital technologies, in particular 3D printing and the Internet of Things, create new ways to rethink the communication of data and bespoke rewarding of individuals achieving activity goals. This has the potential to redirect current systems towards more effective outcomes. Through parametric CAD systems, linked to activity data with algorithms, physical rewards can be produced that represent data in more abstract, tangible ways, allowing people to attach stories to their activity and customise the characters and objects over time. As 3D printing becomes increasingly accessible and well understood by younger generations, and materials such as chocolate and biodegradable materials become commonplace, physical rewards will be able to be developed in ever more engaging ways, well beyond the scope of a flat, digital interface no matter how complex the data it provides. In addition, as the younger generations transition into adult-hood with an evolved understanding of the relationship between people and products, combined with the democratization of making and communication enabled by 
digital technologies, involving them in the integration of the digital and physical will become easier, and more ambitious outcomes increasingly possible.

\section{References}

Ahluwalia, Pal, and Toby Miller. 2014. "The prosumer.” Social Identities 20 (4-5):259-61. doi: 10.1080/13504630.2015.1004830.

Baranowski, Tom, and Leslie Frankel. 2012. "Let's Get Technical! Gaming and Technology for Weight Control and Health Promotion in Children." Childhood Obesity 8 (1):347. doi: http://dx.doi.org/10.1089/chi.2011.0103.

Consolvo, Sunny, David W. McDonald, Tammy Toscos, Mike Y. Chen, Jon Froehlich, Beverly Harrison, Predrag Klasnja, et al. 2008. "Activity sensing in the wild: a field trial of ubifit garden." In Proceedings of the SIGCHI Conference on Human Factors in Computing Systems, 1797-806. Florence, Italy: ACM.

Fleischmann, Katja. 2015. “The Democratisation of Design and Design Learning: How Do We Educate The Next-Generation Designer.” International Journal of Arts \& Sciences 8 (6):101-8.

Gouveia, Rúben, Sergio Barros, and Evangelos Karapanos. 2014. 'Understanding Users' Disengagement with Wearable Activity Trackers." In Proceedings of the 2014 Workshops on Advances in Computer Entertainment Conference, 1-3. Funchal, Portugal: ACM.

Greenfield, Adam. 2006. Everyware: The Dawning Age of Ubiquitous Computing. Berkeley, California USA: New Riders.

Hajkowicz, S. 2015. Global Megatrends: Seven patterns of change shaping our future. Melbourne, Vitoria: CSIRO Publishing.

Hamari, J., J. Koivisto, and H. Sarsa. 2014. Does Gamification Work? -- A Literature Review of Empirical Studies on Gamification. Paper presented at the 2014 47th Hawaii International Conference on System Sciences, 6-9 Jan. 2014.

Hinyard, Leslie J., and Matthew W. Kreuter. 2007. "Using Narrative Communication as a Tool for Health Behavior Change: A Conceptual, Theoretical, and Empirical Overview." Health Education \& Behavior 34 (5):777-92. doi: $10.1177 / 1090198106291963$.

Horejsi, Martin. 2014. “Teaching STEM with a 3D Printer.” The Science Teacher 81 (4):10. 
Hoy, Matthew B. 2016. "Personal Activity Trackers and the Quantified Self." Medical Reference Services Quarterly 35 (1):94-100. doi: 10.1080/02763869.2016.1117300.

Kilpatrick, Marcus, Edward Hebert, and Dee Jacobsen. 2002. "Physical Activity Motivation:

A Practitioner's Guide to Self-Determination Theory." Journal of Physical

Education, Recreation \& Dance 73 (4):36-41. doi:

10.1080/07303084.2002.10607789.

Krishna, Golden. 2015. The Best Interface is No Interface: the Simple Path to Brilliant Technology. USA: New Riders.

Ledger, Dan. 2016. "Inside Wearables - Part 3: The rocky path towards personalized, insightful wearables." In Inside Wearables.

Ledger, Dan, and Daniel McCaffrey. 2014. "Inside Wearables: How the Science of Human Behavior Change Offers the Secret to Long-Term Engagement.” In Inside Wearables.

Lin, James J., Lena Mamykina, Silvia Lindtner, Gregory Delajoux, and Henry B. Strub. 2006. "Fish'n'Steps: Encouraging Physical Activity with an Interactive Computer Game." In UbiComp 2006: Ubiquitous Computing: 8th International Conference, UbiComp 2006 Orange County, CA, USA, September 17-21, 2006 Proceedings, edited by Paul Dourish and Adrian Friday, 261-78. Berlin, Heidelberg: Springer Berlin Heidelberg. Lou, Nicole. 2016. "Rise of the Makerspace.” Popular Science, 2016 March-April, 88.

Nicholson, Scott. 2012. “A User-Centered Theoretical Framework for Meaningful Gamification." In Games+Learning+Society 8.0, 7. Madison, WI.

Norman, Don. 2016. “The Paradox of Wearable Technologies.” MIT Technology Review, Accessed 13 December. https://www.technologyreview.com/s/517346/the-paradoxof-wearable-technologies/.

Novak, James, and Jennifer Loy. 2017a. Responsive Tangible Rewards in Physical Activity Gamification. Paper presented at the Fourth International Conference on Design4Health 2017, Melbourne Cricket Ground, Melbourne, Victoria, Australia.

Novak, James, and Jennifer Loy. 2017b. "Digital Technologies and 4D Customized Design: Challenging Conventions with Responsive Design." In Handbook of Research on Human Development in the Digital Age, edited by Valerie C. Bryan, Ann T. Musgrove and Jillian R. Powers, 403-26. Hershey, PA, USA: IGI Global.

Piwek, Lukasz, David A. Ellis, Sally Andrews, and Adam Joinson. 2016. "The Rise of Consumer Health Wearables: Promises and Barriers." PLoS Medicine 13 (2). doi: 10.1371/journal.pmed.1001953 
Strava. (2017). 2017 in Stats. Retrieved from https://blog.strava.com/2017-in-stats/

Qian, Zhenyu Cheryl. 2009. "Design Patterns: Augmenting Design Practice in Parametric CAD Systems.” Ph.D., Simon Fraser University (Canada).

Shugrina, Maria, Ariel Shamir, and Wojciech Matusik. 2015. "Fab Forms: Customizable Objects for Fabrication with Validity and Geometry Caching." In ACM Siggraph 2015. Los Angeles.

Sterling, Bruce. 2005. Shaping Things. Cambridge, Massachusetts: The MIT Press.

Weiser, Mark. 1991. "The Computer for the 21st Century." Scientific American 265 (3):94110.

Weiser, Mark, and John Seely Brown. 1996. "Designing calm technology.” PowerGrid Journal 1 (1):75-85.

Wilson, Lyndal. 2013. "A New Dimension: The Use of 3D Printing in Schools." Independence 38 (2):26, 28-32.

World Health Organisation. 2018. "Obesity and Overweight.” World Health Organisation, Accessed 16 April. http://www.who.int/mediacentre/factsheets/fs311/en/.

Zuckerman, Oren, and Ayelet Gal-Oz. 2014. "Deconstructing Gamification: Evaluating the Effectiveness of Continuous Measurement, Virtual Rewards, and Social Comparison for Promoting Physical Activity.” Personal and Ubiquitous Computing 18 (7):170519. doi: 10.1007/s00779-014-0783-2.

\section{Biographies}

Dr James Novak is a postdoctoral researcher in Product Design and Design for Additive Manufacturing at the University of Technology Sydney, Australia. He has a particular research interest in 4D products and the use of additive manufacturing to improve/support human health.

Dr Jennifer Loy is Professor of Product Design \& Design for Additive Manufacturing at the University of Technology Sydney and research theme leader for Transformative Technologies across Design, Architecture and Building and the School of Engineering. She is also an Adjunct Professor at the Menzies Health Institute in Chronic Conditions and was a 
founder member of the Gold Coast Orthopaedics and Musculoskeletal Health Research Alliance. 


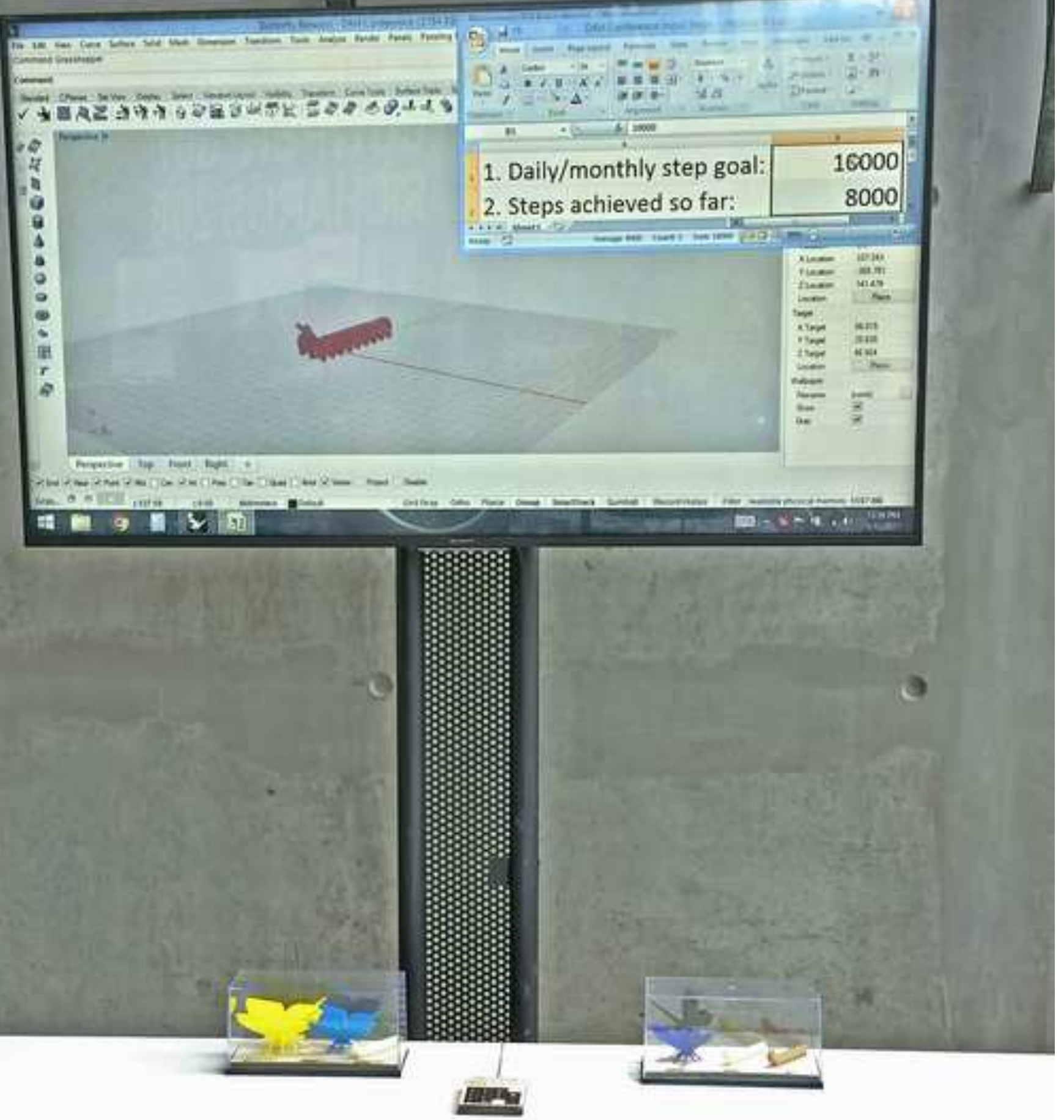




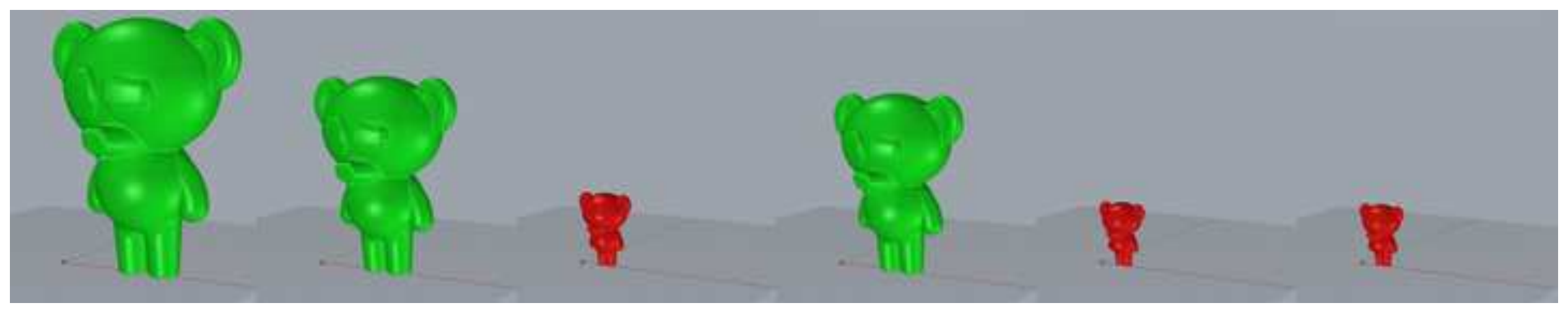

Figure 3 
Figure (high resolution)
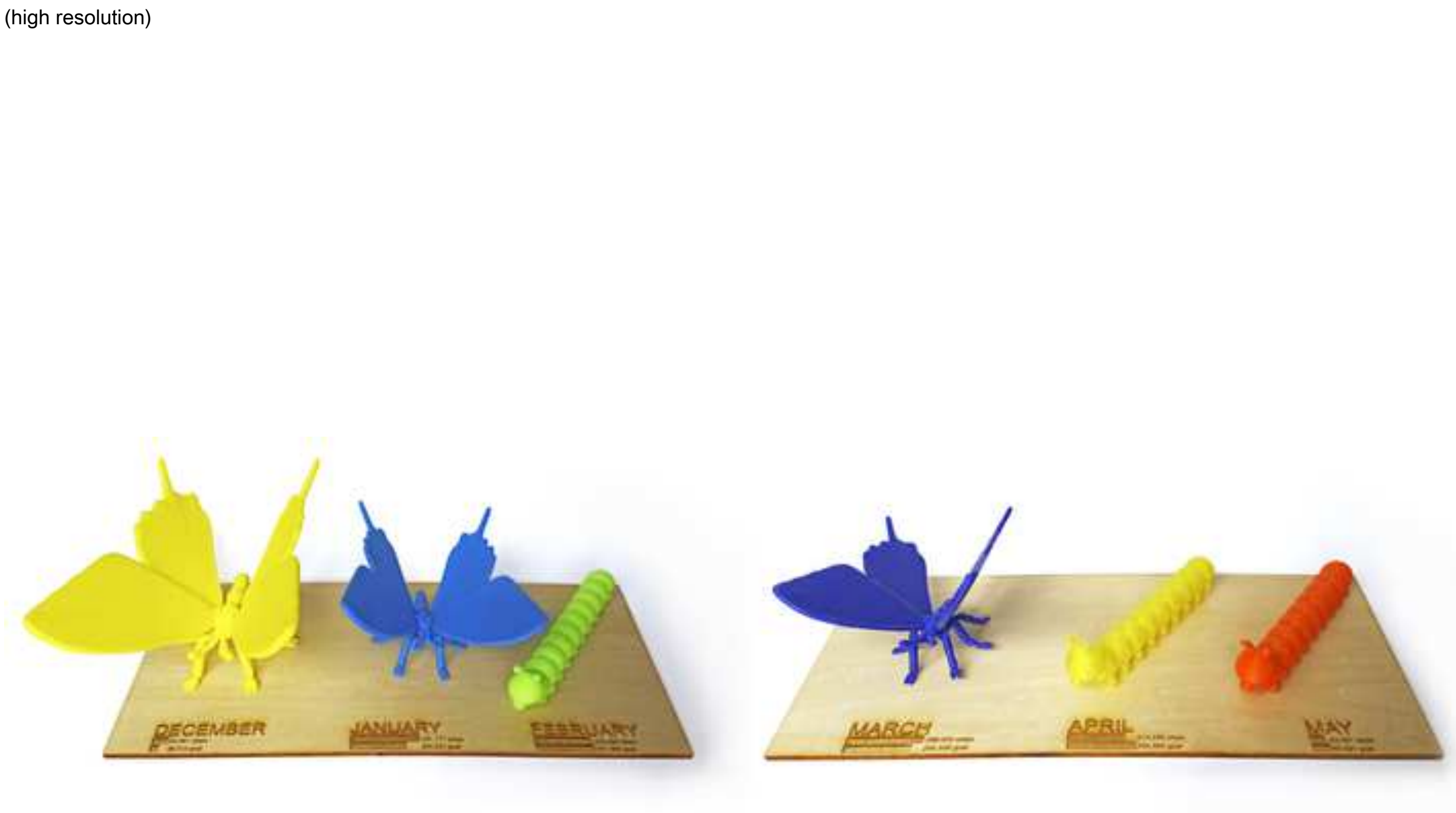

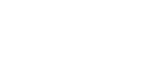
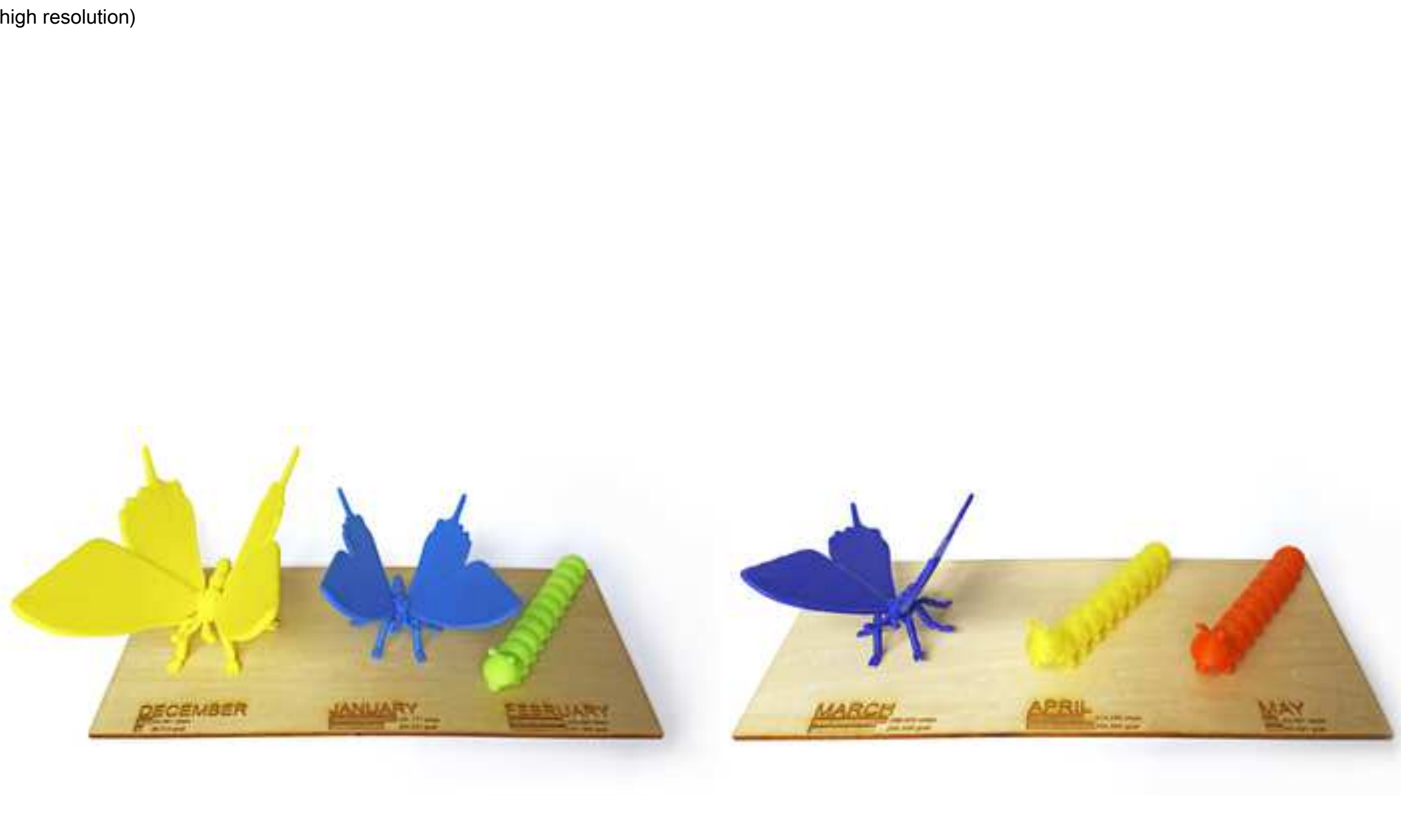

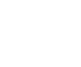
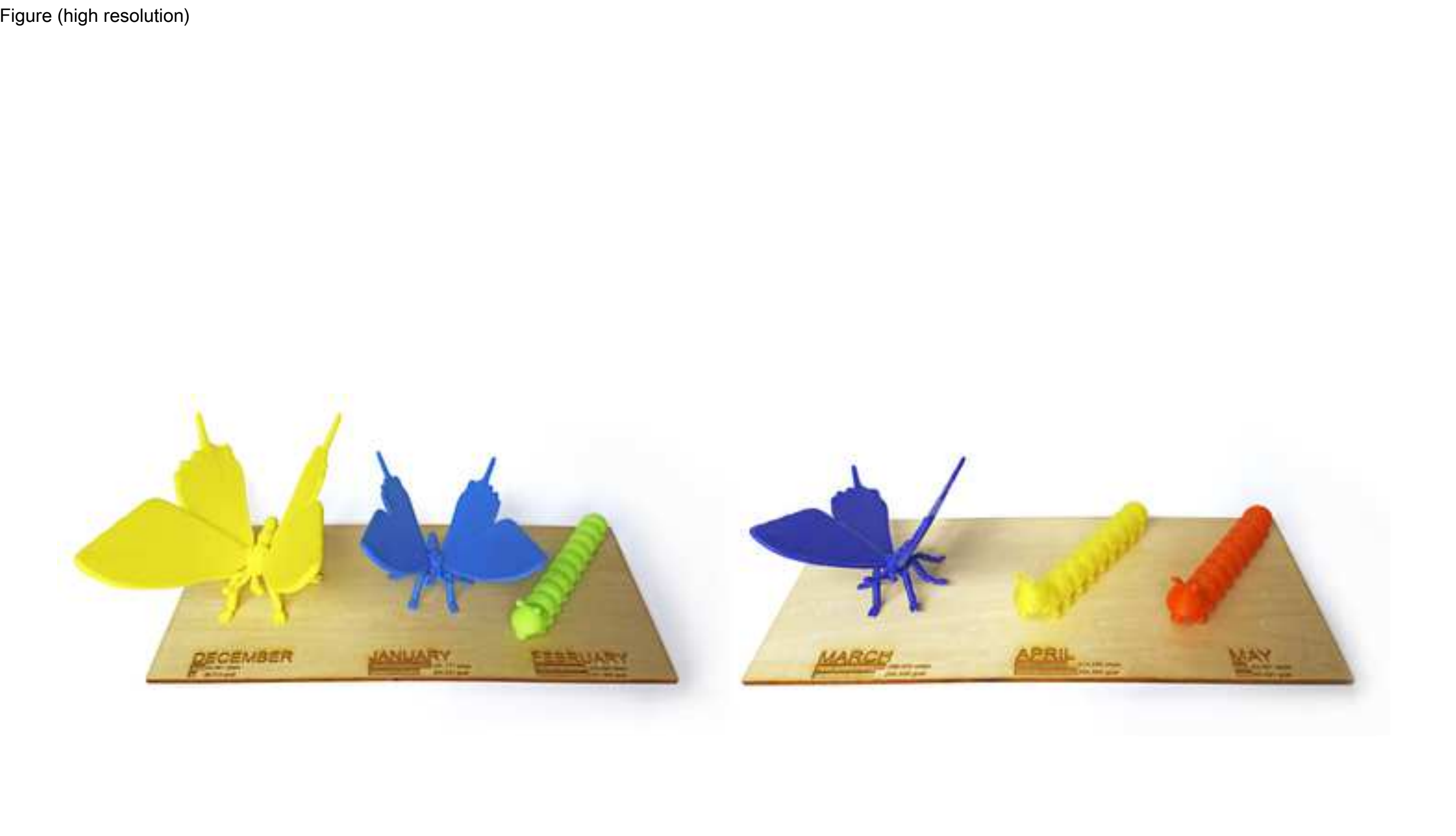

.
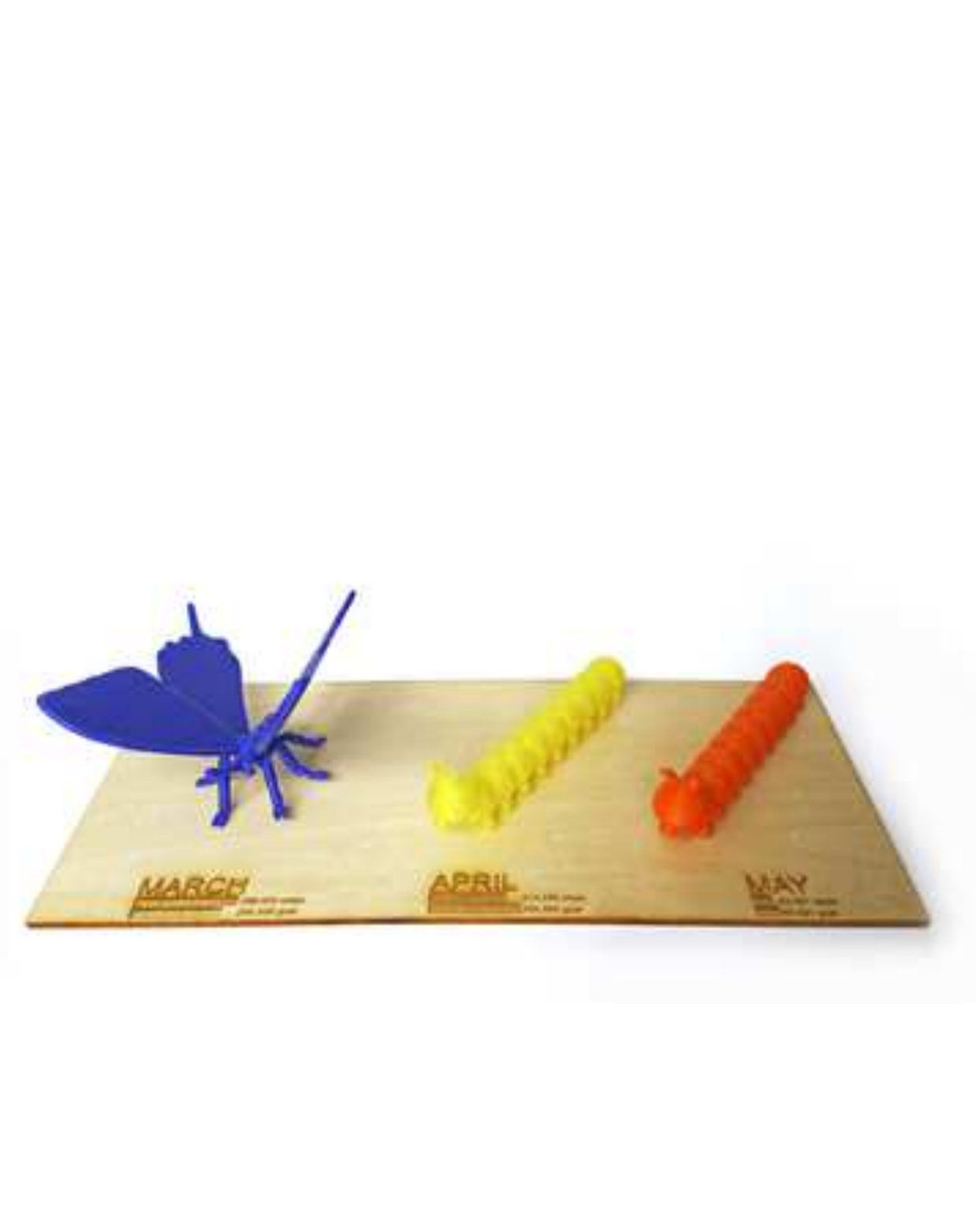

(1)

.

.

.

.

( 


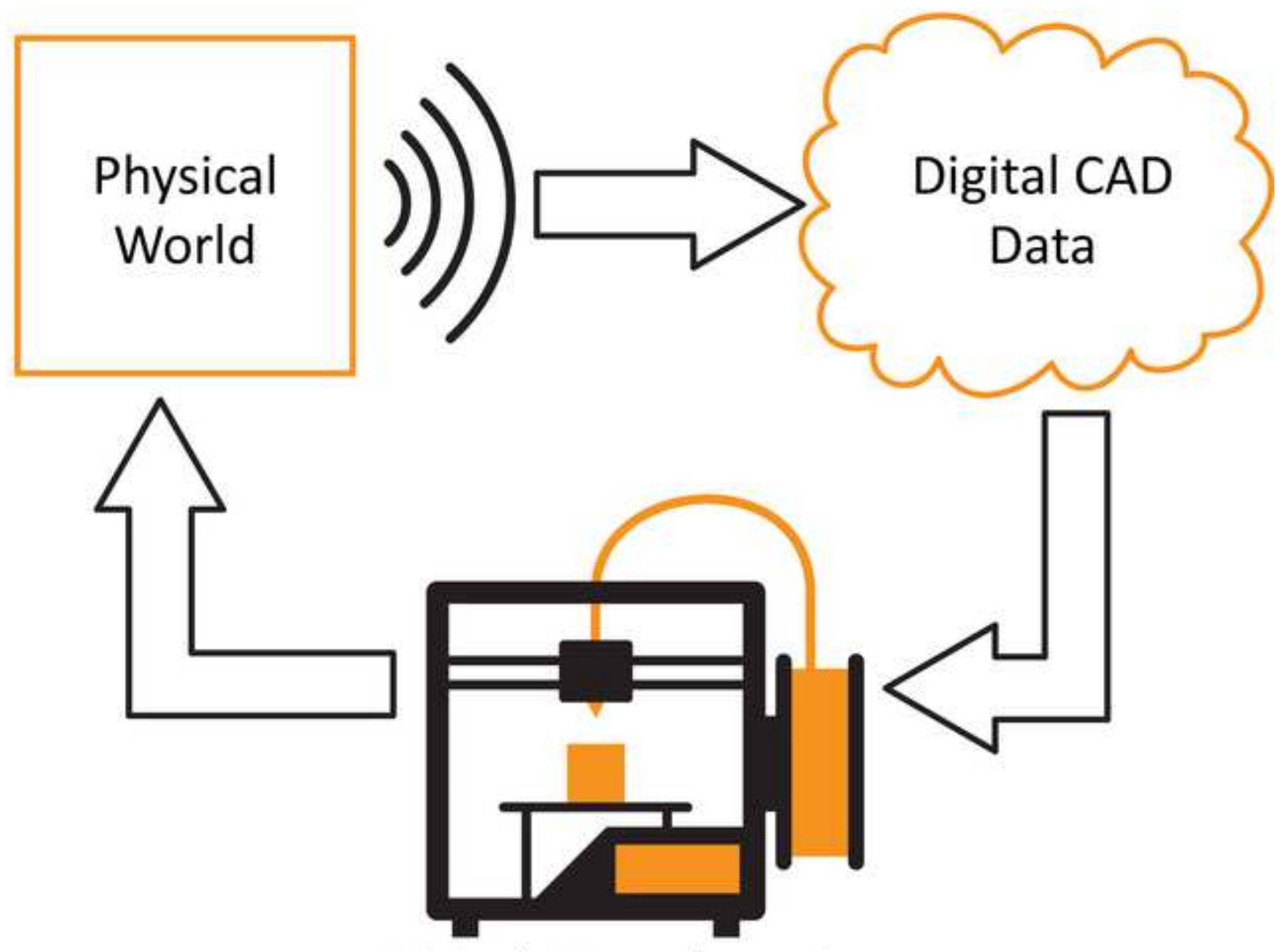

Digital Manufacturing 


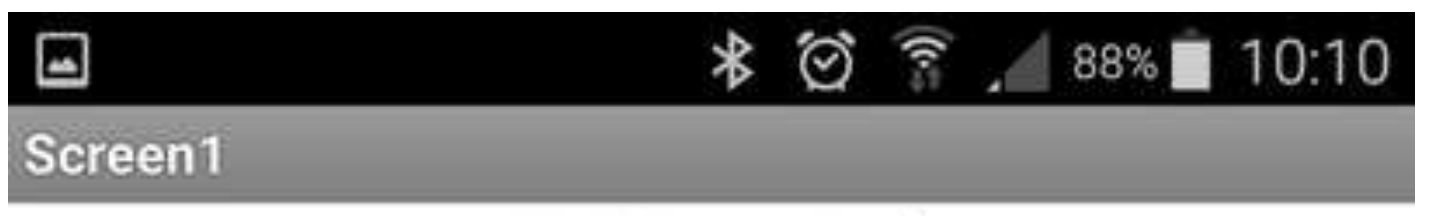

\section{Not Connected}

\section{Connect to PEZ via Bluetooth}

\section{Set Step Goal: 50}

\section{Start Counting}

\section{Stop Counting Steps}

Steps:

RESET 


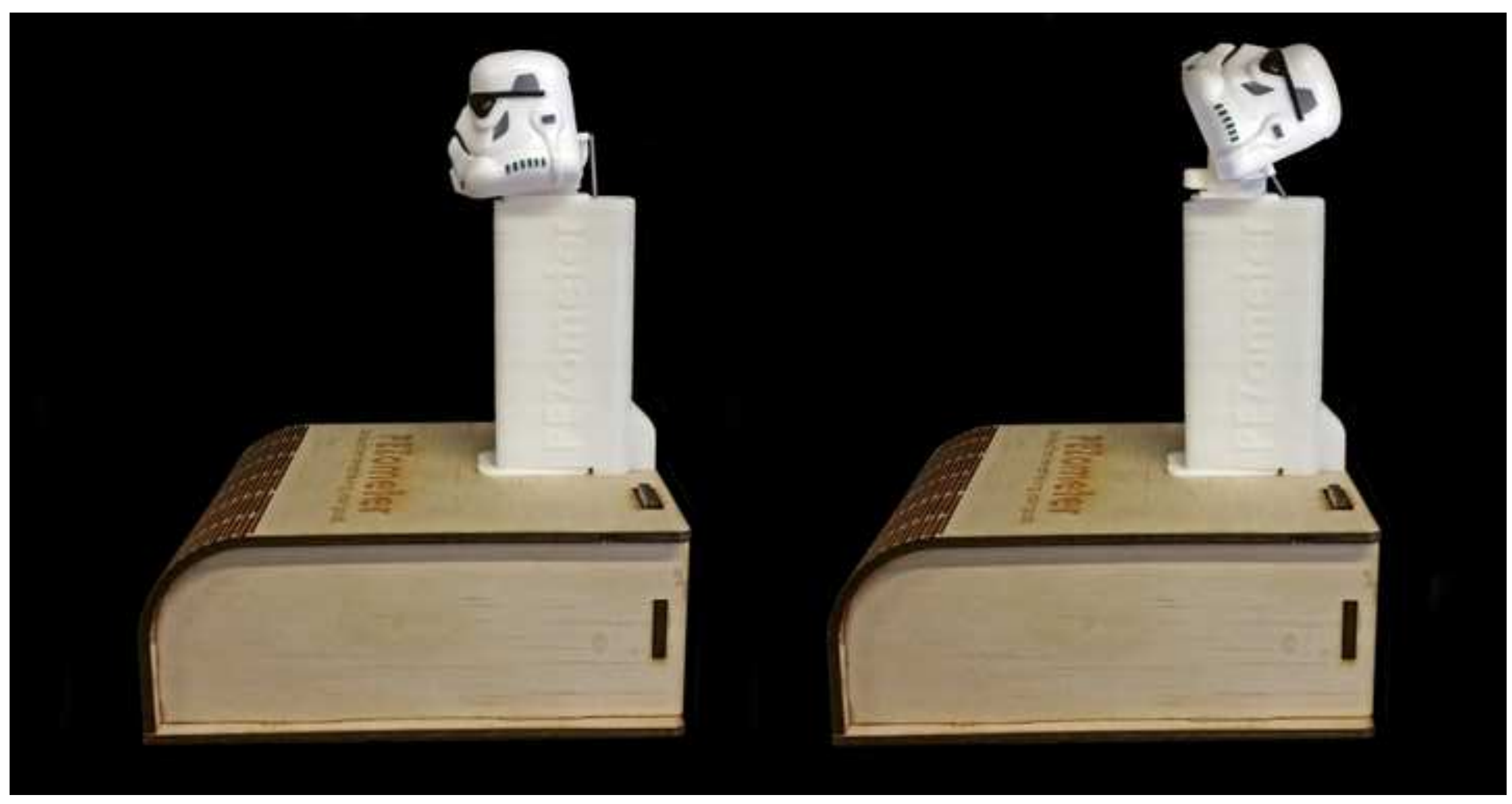

